\title{
TWO-PHOTON RESONANT THIRD HARMONIC GENERATION IN Hg
}

\author{
E. KOUDOUMAS ${ }^{+}$and T. EFTHIMIOPOULOS* \\ ${ }^{+}$Foundation for Research and Technology_Hellas, Institute of Electronic \\ Structure and Laser, (FORTH, IESL) *Physics Department, University of Crete \\ Iraklion, Crete, Greece, 71409.
}

(Received 8 June, 1992)

Third-harmonic generation in $\mathrm{Hg}$ vapors, using the two-photon resonance $6^{1} \mathrm{~S}_{0}-8^{1} \mathrm{~S}_{0}$ transition is theoretically and experimentally investigated. Calculations of the $\mathrm{Hg}$ contribution to the phase mismatch are presented and the effects of the absorption of the radiation, the population transfer, the saturation, and the ionization on the efficiency of the system are considered. It is shown that the population transfer is the dominant mechanism affecting the efficiency.

KEY WORDS: Third harmonic generation, two-photon resonance, phase matching, population transfer.

\section{INTRODUCTION}

Resonant and nonresonant third-harmonic generation and frequency mixing have already been investigated in detail, experimentally and theoretically and several atomic media, such as metal vapors and rare gases, have been used for generation of radiation that covers the region from $200 \mathrm{~nm}$ to well down in the XUV region. ${ }^{1-14}$ In most of the cases enhancement of the efficiency was achieved with two-photon resonances which increases the non-linear susceptibility, avoiding pump depletion at the same time. , $2,4,9-14$ Additional near resonances to broad autoionizing states resulted in further enhancement of the efficiency. ${ }^{15}$

As the generated intensity depends on the input intensity third power and the phase-matching between the third harmonic (TH) and the fundamental frequencies, ${ }^{5,16,17}$ tight focusing of the input beam ${ }^{4,5}$ and two-component systems ${ }^{3-5}$ were used to optimize the mixing processes. High intensities, however, not only improved the generated intensity, but were accompanied by limiting processes, resulting in saturation. Processes, such as depletion of the fundamental due to two-photon absorption, population transfer to the resonant state and non-linearity in the refractive index caused by second order Kerr effect ${ }^{5-12}$ set an upper limit to the conversion efficiency.

In this study we present experimental results together with calculations of the third-harmonic generation in $\mathrm{Hg}$, using a two-photon resonance to the $8^{1} \mathrm{~S}_{0}$ state. $\mathrm{Hg}$ was chosen for its high ionization potential $(10.4 \mathrm{eV})$, and the large number of 
autoionizing states ${ }^{18-20}$ available for the generation of radiation of very short wavelengths. The state $8^{1} \mathrm{~S}_{0}$ was chosen as the two-photon resonant state because the autoionizing states can be reached through this state, with available visible or near-UV photons. Figure 1 shows a simplified energy level diagram of $\mathrm{Hg}$ relevant to this work.

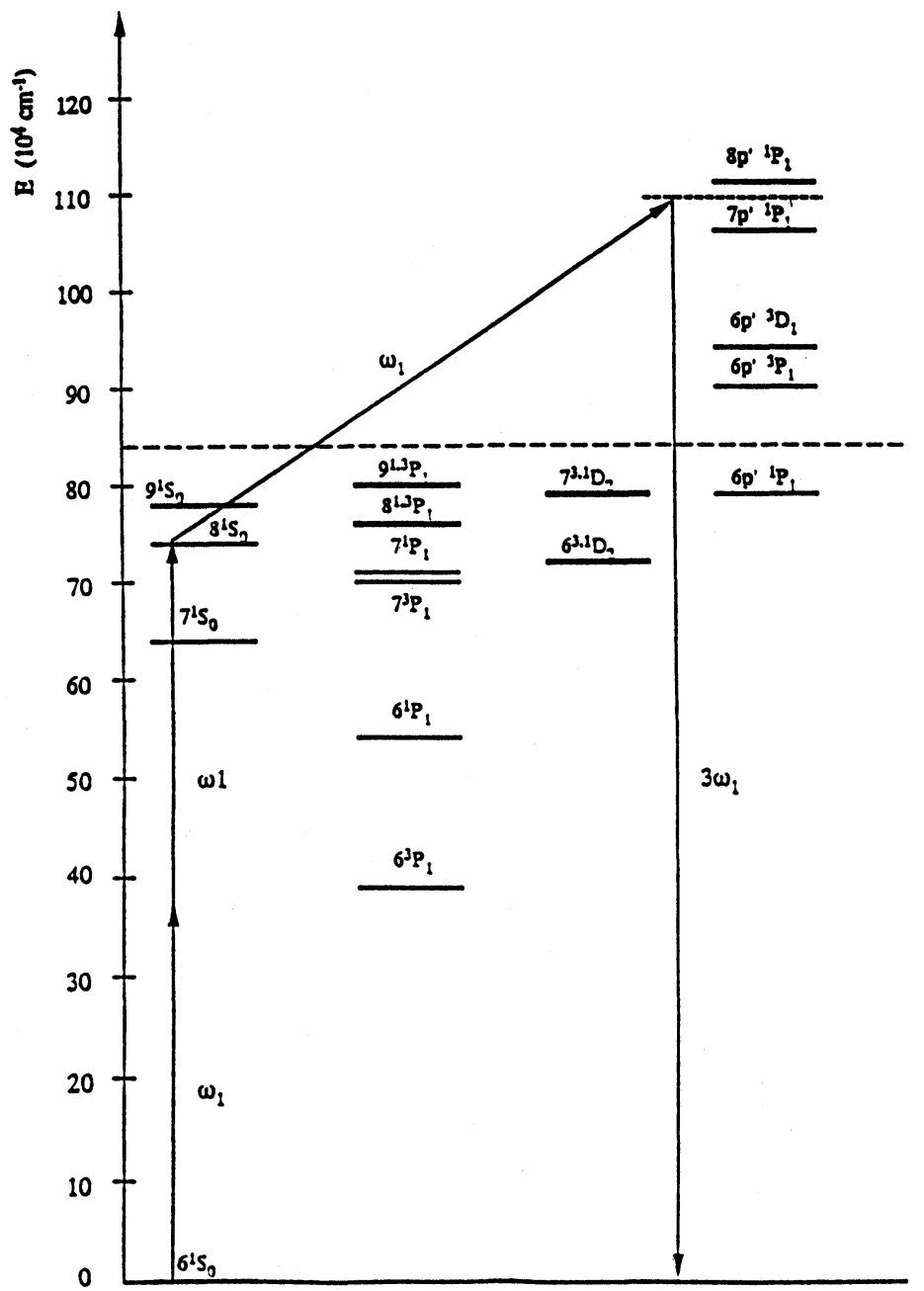

Figure 1 Simplified energy level diagram of $\mathrm{Hg}$.

Several authors ${ }^{4,12-14}$ have reported non-linear mixing in $\mathrm{Hg}$ vapor generating radiation that covers the region $87.5-130 \mathrm{~nm}$. However, there are some aspects of the problem which have not been dealt with. In this paper we do calculations concerning the wavevector mismatch, including contributions from the autoionizing states. We investigate the effects on the phase mismatch of the population transfer to the resonant state, the AC Stark shift, and the ionization. Finally we provide a 
working guideline for the operation of an efficient system for the users of such sources.

\section{EXPERIMENTAL}

An excimer laser (LUMONICS HYPEREX-400) operated at $5 \mathrm{~Hz}$, providing $20 \mathrm{MW}$ pulses of $20 \mathrm{~ns}$ duration was used to pump a dye laser (LUMONICS HYPERDYE300 ). The dye laser radiation $\omega$ with $2 \mathrm{MW}$ pulses of $17 \mathrm{~ns}$ duration and $0.09 \mathrm{~cm}^{-1}$ linewidth, was frequency doubled in a KDP crystal (INRAD) yielding a peak power of $200 \mathrm{~kW}$. The doubled frequency radiation $\omega_{1}=2 \omega$, separated from the fundamental with a prism, was focused, with a $20 \mathrm{~cm}$ focal length lens, through a quartz window, into a pyrex heat pipe, similar to the one described by Herman et al, ${ }^{4}$ containing $\mathrm{Hg}$. He buffer gas of equal or higher pressure together with water-cooled jackets were used to confine the vapor in a zone $\mathrm{L}$ of approximately $10 \mathrm{~cm}$ length. A needle valve was used to control the pressure of $\mathrm{He}$. The $\mathrm{He}$ gas was passed through a liquid nitrogen trap in order to remove impurities. Because the wavelength of the third harmonic, which we studied, is well below the LiF cutoff (104 nm) no exit window could be used. Instead a $100 \mu \mathrm{m}$ pinhole and differential pumping were employed, allowing a He flow of $16 \mathrm{ml} / \mathrm{min}^{21}$ and a pressure drop down to $10^{-5} \mathrm{mbar}$ in the $0.2 \mathrm{~m}$ focal length vacuum monochromator (McPherson 34), which was adequate to operate the electron multiplier (Hamamatsu R546) used for detection. The transmission of the light through the pinhole was low (5\%) but the small pinhole helped to avoid any pressure gradient in the cell. Calibration of the wavelength was done using the optogalvanic effect. ${ }^{22}$ The output signal of the electron multiplier was fed in a boxcar averager (SRS, SR250) and individual measurement points were the average of 100 laser shots.

\section{RESULTS AND DISCUSSION}

The frequency $\omega_{1}$ was tuned to the two-photon resonance ${ }^{1} \mathrm{~S}_{0}-8^{1} \mathrm{~S}_{0}\left(\lambda_{1}=268.8 \mathrm{~nm}\right)$ (Figure 1) with the third photon reaching a level $665 \mathrm{~cm}^{-1}$ from the nearest autoionizing state $8 p^{\prime}{ }^{1} P_{1}$. The recorded TH intensity as a function of the $2 \omega_{1}$ photon energy is shown in Figure 2. The conversion efficiency was estimated to be approximately $10^{-7}$, resulting in $10^{8}$ photons per pulse (corresponding to $13 \mathrm{~mW}$ ). In the following sections we discuss results concerning the calculation of the wavevector mismatch, the influence of the experimental parameters on the process, and the effect of the population transfer, the AC-Stark shift and the ionization on the wavevector mismatch.

\subsection{Calculation of the Wavevector Mismatch}

For a tightly focused incident beam, such as the one used in these experiments, the generated intensity in third harmonic generation is proportional to the phase-matching 


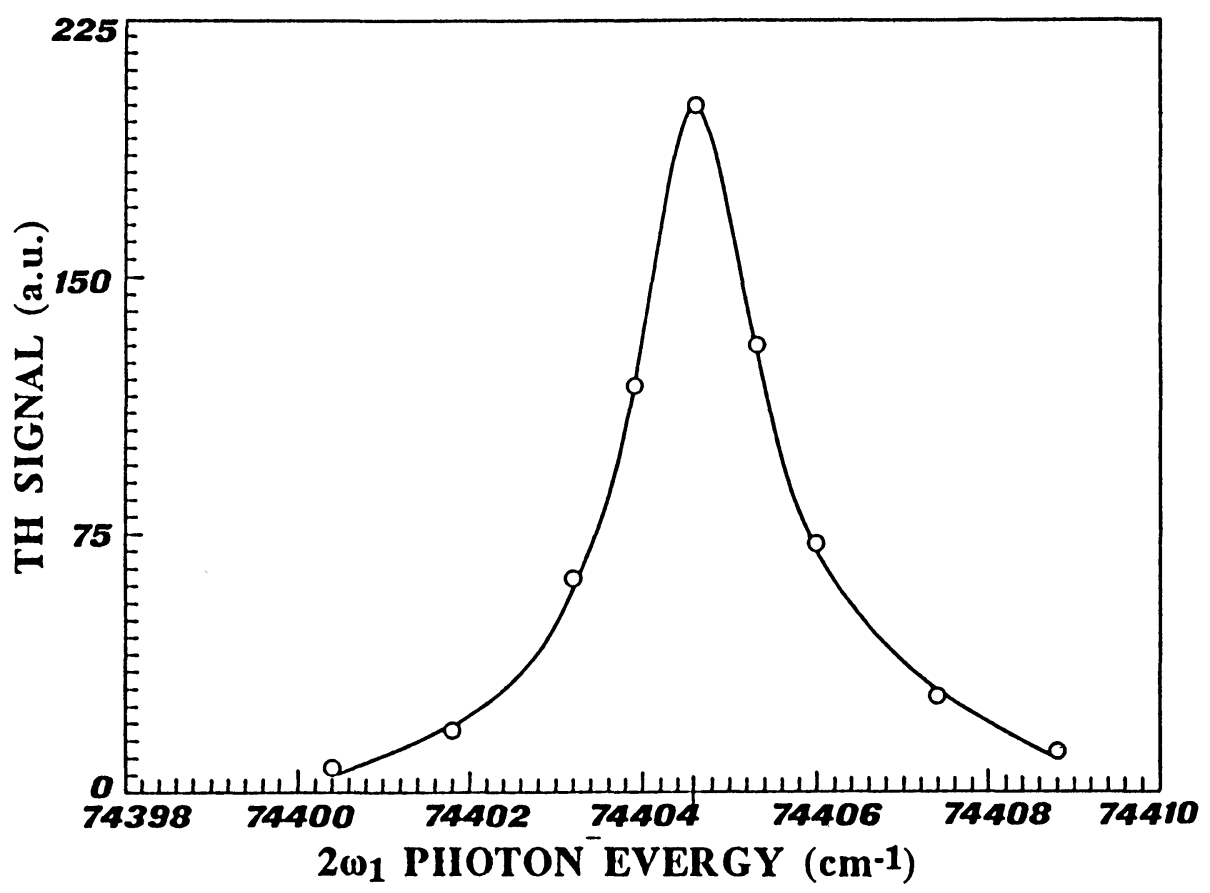

Figure 2 TH intensity as a function of the $2 \omega_{1}$ photons energy. Maximum enhancement appears on resonance at the $8^{1} \mathrm{~S}_{0}$ state, $\left(74404.6 \mathrm{~cm}^{-1}\right.$, Moore $\left.{ }^{27}\right)$. The experimental parameters are: $\mathrm{I}=3.810^{8} \mathrm{~W} / \mathrm{cm}^{2}$, $\mathrm{N}_{\mathrm{Hg}}=3.510^{17} \mathrm{~cm}^{-3}, \mathrm{~N}_{\mathrm{He}}=7.510^{17} \mathrm{~cm}^{-3}$.

factor F. In general, this factor is a function of the confocal parameter $b$ of the input laser beam and of the mismatch $\Delta \mathrm{k}$ between the wavevectors of the generated and the fundamental frequencies. The wavevector mismatch in the case of TH generation is given by $\Delta \mathrm{k}=6 \pi v_{1}\left(\mathrm{n}_{3}-\mathrm{n}_{1}\right)$, where $v_{1}$ is the input photon energy in $\mathrm{cm}^{-1}$, and $\mathrm{n}_{3}, \mathrm{n}_{1}$ are the linear refractive indices at the harmonic and the fundamental wavelengths. For the calculation of the wavevector mismatch, we need to know the $\mathrm{n}_{3}$ and $\mathrm{n}_{1}$ values, which, for atoms in the ground state and neglecting any non-linear contributions, are generally given ${ }^{23}$ by $n_{j}-1=2 \pi N \alpha_{g}{ }^{\prime}\left(\omega_{j}\right)$, where $\alpha_{g}^{\prime}\left(\omega_{j}\right)$ is the real part of the ground state $(\mathrm{g})$ linear polarizability. The contribution of the bound states and the continuum on the value of $\alpha_{\mathrm{g}}{ }^{\prime}\left(\omega_{\mathrm{j}}\right)$ can be estimated using known equations, ${ }^{6,7,23}$ while in the case of autoionizing states the contribution can be calculated separately using an equation reported by Alber and Zoller. ${ }^{24}$

For the calculation of the exact value of the $\mathrm{Hg}$ contribution to the wavevector mismatch $(\mathrm{C}=\Delta \mathrm{k} / \mathrm{N})$ we need to know all the matrix elements for the transitions in $\mathrm{Hg}$. Unfortunately for the $\mathrm{Hg}$ atom few data exist concerning oscillator strengths and matrix elements, especially for transitions between excited states. Experimental oscillator strengths for transitions between the ground state and excited states up to the state $13^{1} \mathrm{P}_{1}$, have been reported by Smith et al. ${ }^{23}$ These oscillator strengths, the calculated matrix elements ${ }^{17}$ and the corresponding energy states, given by Moore, ${ }^{19}$ are presented in Table 1 . The oscillator strengths for states above the $13^{1} P_{1}$ are 
expected to be minimal (because their magnitude drops with increased principal quantum number) and their contribution to the wavevector mismatch is very low.

Table 1 Experimental oscillator strengths, reported by Smith and Alford, ${ }^{23}$ matrix elements and energy level spacing, given by Moore, ${ }^{19}$ for the transitions $6^{1} S^{0}-n^{1,3} P_{1}, n=6-13$.

\begin{tabular}{llll}
\hline Upper level & Energy spacing $\mathrm{cm}^{-1}$ & Oscillator strength & Matrix element $10^{-9} \mathrm{~cm}$ \\
\hline $6^{3} \mathrm{P}_{1}$ & 39412 & 0.024 & 1.3675 \\
$6 \mathrm{P}^{1} \mathrm{P}_{1}$ & 54069 & 1.15 & 8.0821 \\
$7^{3} \mathrm{P}_{1}$ & 69662 & $<10^{-5}$ & 0.0212 \\
$7^{1} \mathrm{P}_{1}$ & 71295 & 0.02 & 0.9279 \\
$8^{3} \mathrm{P}_{1}$ & 76467 & 0.0014 & 0.2370 \\
$8^{1} \mathrm{P}_{1}$ & 76863 & 0.01 & 0.6322 \\
$6 \mathrm{P}^{\prime}{ }^{1} \mathrm{P}_{1}$ & 78813 & 0.15 & 2.4175 \\
$9^{3} \mathrm{P}_{1}$ & 79413 & 0.0055 & 0.4612 \\
$9^{1} \mathrm{P}_{1}$ & 79964 & 0.07 & 1.6400 \\
$10^{3} \mathrm{P}_{1}$ & 80917 & 0.0018 & 0.2614 \\
$10^{1} \mathrm{P}_{1}$ & 81154 & 0.0155 & 0.7659 \\
$11^{3} \mathrm{P}_{1}$ & 81812 & 0.0011 & 0.4329 \\
$11^{1} \mathrm{P}_{1}$ & 81943 & 0.0050 & 0.4329 \\
$12^{3} \mathrm{P}_{1}$ & 82379 & 0.0008 & 0.1727 \\
$12^{1} \mathrm{P}_{1}$ & 82464 & 0.0023 & 0.2927 \\
$13^{3} \mathrm{P}_{1}$ & 82795 & 0.0005 & 0.1362 \\
$13^{1} \mathrm{P}_{1}$ & 82824 & 0.0011 & 0.2020 \\
\hline
\end{tabular}

Oscillator strengths for transitions between the ground state and the autoionizing states are not well known for $\mathrm{Hg}$. Only for the transition $6^{1} \mathrm{~S}_{0}-6 \mathrm{p}^{\prime}{ }^{3} \mathrm{P}_{1}$, Lincke et al ${ }^{25}$ have calculated a value of $\mathrm{f}=0.53$, using the equation $\mathrm{f}=\left(\mathrm{mc}^{2} / 2 \mathrm{e}^{2}\right) \sigma_{\mathrm{a}} \Gamma\left(\mathrm{q}^{2}-1\right)$, where $\mathrm{f}$ is the oscillator strength, $\sigma_{\mathrm{a}}$ is the photoionization cross-section for the part of the continuum that interacts with that state, $\Gamma$ is the autoionization linewidth in $\mathrm{cm}^{-1}$, and $\mathrm{q}$ is the corresponding Fano parameter. The values of the $\mathrm{q}$ and $\Gamma$ parameters of the $6 \mathrm{p}^{\prime}{ }^{3} \mathrm{D}_{1}$ state of $\mathrm{Hg}$ have been reported by Schafer et al. ${ }^{26}$ For the states $7 \mathrm{p}^{\prime}$ ${ }^{1} \mathrm{P}_{1}, 8 \mathrm{p}^{\prime}{ }^{1} \mathrm{P}_{1}$ we estimated the oscillator strengths by fitting the function $(\mathrm{q}+\mathrm{e})^{2} / 1+\mathrm{e}^{2}$ (where $\mathrm{e}=2\left[\mathrm{E}-\mathrm{E}_{\mathrm{r}}\right] / \Gamma, \mathrm{E}_{\mathrm{r}}$ being the energy of the state) to the photoionization data reported by $\mathrm{Brehm}^{20}$ and by using the cross-section values reported by Berkowitz. ${ }^{18}$ The results together with the already reported information are presented in Table 2 , where $\sigma_{\max }$ is the maximum absorption cross section and $\sigma_{\mathrm{b}}$ is the photoionization cross-section for the part of the continuum that does not interact with the state and all the other parameters have been defined above. In the case of the $8 \mathrm{p}^{\prime}{ }^{1} \mathrm{P}_{1}$ state there is an uncertainty in the calculation of the q parameter, resulting in uncertainty in the $\sigma_{\mathrm{a}}$. However the oscillator strength depends on the product $\left(\mathrm{q}^{2}-1\right) \sigma_{\mathrm{a}}$, which is approximately equal to the $\sigma_{\max }$, for high q values, and this is known with better accuracy.$^{18}$ For higher autoionizing states the fitting can not be done accurately, but because the TH energy $\left(111606 \mathrm{~cm}^{-1}\right)$ is between the energy of the states $7 \mathrm{p}^{\prime}{ }^{1} \mathrm{P}_{1}$ and $8 \mathrm{p}^{\prime}{ }^{1} \mathrm{P}_{1}$, it is the contribution of these states and the contribution of the $6 \mathrm{p}^{\prime}{ }^{3} \mathrm{P}_{1}$ and $6 \mathrm{p}^{\prime}{ }^{3} \mathrm{D}_{1}$ states (due to their large matrix element) which dominates. Also the 
states above the $8 \mathrm{p}^{\prime}{ }^{1} \mathrm{P}_{1}$ are expected to have low contribution because of their high principal quantum number (see Table 2).

Table 2 Some $\mathrm{Hg}$ autoionizing states parameters. a: Lincke and Stredele, ${ }^{25}$ b: Schafers et al ${ }^{26}$ c: Berkowitz ${ }^{18} \mathrm{~d}$ : Moore ${ }^{19}$ Other parameters are calculated from data reported by Brehm. ${ }^{20}$

\begin{tabular}{lllllllll}
\hline $\begin{array}{l}\text { Upper } \\
\text { state }\end{array}$ & $q$ & $\Gamma \mathrm{cm}^{-1}$ & $\begin{array}{l}\text { Energy } \\
\text { of state } \\
c m-1\end{array}$ & $\begin{array}{l}\sigma_{\max } \\
10^{-18} \\
\mathrm{~cm}^{2}\end{array}$ & $\begin{array}{l}\sigma_{a} 10^{-18} \\
\mathrm{~cm}^{2}\end{array}$ & $\sigma_{b} \mathrm{~cm}^{2}$ & $f$ & $\begin{array}{l}\text { matrix } \\
\text { element } \\
10-9 \mathrm{~cm}\end{array}$ \\
\hline $6 \mathrm{P}^{\prime}{ }^{3} \mathrm{P}_{1}$ & $11^{\mathrm{a}}$ & $1645^{\mathrm{a}}$ & $88760^{\mathrm{d}}$ & $193^{\mathrm{c}}$ & $1.78^{\mathrm{a}}$ & $\sim 10^{-20}$ & 0.53 & 4.282 \\
$6 \mathrm{P}^{\prime}{ }^{3} \mathrm{D}_{1}$ & $6^{\mathrm{b}}$ & $177^{\mathrm{b}}$ & $93737^{\mathrm{d}}$ & $90^{\mathrm{c}}$ & 2.64 & $\sim 10^{-20}$ & 0.030 & 1.586 \\
$7 \mathrm{P}^{\prime}{ }^{1} \mathrm{P}_{1}$ & 6.5 & 129 & $105881^{\mathrm{d}}$ & $200^{\mathrm{c}}$ & 4.88 & $\sim 10^{-20}$ & 0.047 & 1.168 \\
$8 \mathrm{P}^{\prime}{ }^{1} \mathrm{P}_{1}$ & $10 \pm 2$ & 77 & $112272^{\mathrm{d}}$ & $75^{\mathrm{c}}$ & $0.9 \pm 0.3$ & $\sim 10^{-20}$ & 0.011 & 0.549 \\
\hline
\end{tabular}

The calculation of the $\Delta \mathrm{k}_{\mathrm{Hg}}$ was carried out in the energy region 111590$111620 \mathrm{~cm}^{-1}$, using the reported equations, the matrix element values from tables 1 and 2 and the assumption that all the atoms are in the ground state. Excited states up to the $13^{1} \mathrm{P}^{1}$ state seems to give the largest contribution, because of their oscillator strength. The contribution of the continuum was assumed to be negligible. This assumption is supported by the large difference between the photoionization cross-sections due to the autoionizing states and the one due to the continuum (Table 2). Our calculations gave a linear slowly varying atomic $\mathrm{Hg}$ contribution to the wave-vector mismatch with a value of $\mathrm{C}_{\mathrm{Hg}}=-3.6910^{-17} \mathrm{~cm}^{2}$ for the region of interest. The calculation for the contribution of He, where data reported by Hey et $\mathrm{al}^{27}$ were used, gives a value of $\mathrm{C}_{\mathrm{He}}=3.6810^{-19} \mathrm{~cm}^{2}$ (the He contribution to the phase mismatch is also a linear slowly varying function of the photon energy). This value suggests that the $\mathrm{He}$ buffer gas will give comparable contribution to the $\mathrm{Hg}$ one for a pressure of the order of a few hundred Torr. This is in disagreement with the experimental results of the following section.

\subsection{Experimental Results}

Figure 3 shows the intensity of the TH signal as a function of the number density of $\mathrm{Hg}$. At low number densities the curve is linear with slope 1.6 while saturation appears as the number density increases. According to the calculations presented by Bjorklund ${ }^{16}$ in the case where the number density $\mathrm{N}$ is a free parameter, maximization of the phase matching function $G$ appears at a value of $N=|4 / b C|$, where $b$ is the confocal parameter of the incoming laser beam and $\mathrm{C}$ are the atomic contributions to $\Delta \mathrm{k}(\Delta \mathrm{k}=\mathrm{NC})$. In the case of the $\mathrm{TH}$ generation at $89.6 \mathrm{~nm}$, the number density that maximizes the function $\mathrm{F}$ is calculated to be $410^{16} \mathrm{~cm}^{-3}$ and for the number densities in use, the function $\mathrm{F}$ and the third harmonic signal are expected to decrease with increased $\mathrm{N}$. This is in contrast with the experimental results of Figure 3.

In addition, the absorption of the incoming and the generated radiations should be included. According to data given by Drullinger et $a^{28}$ the one photon absorption 


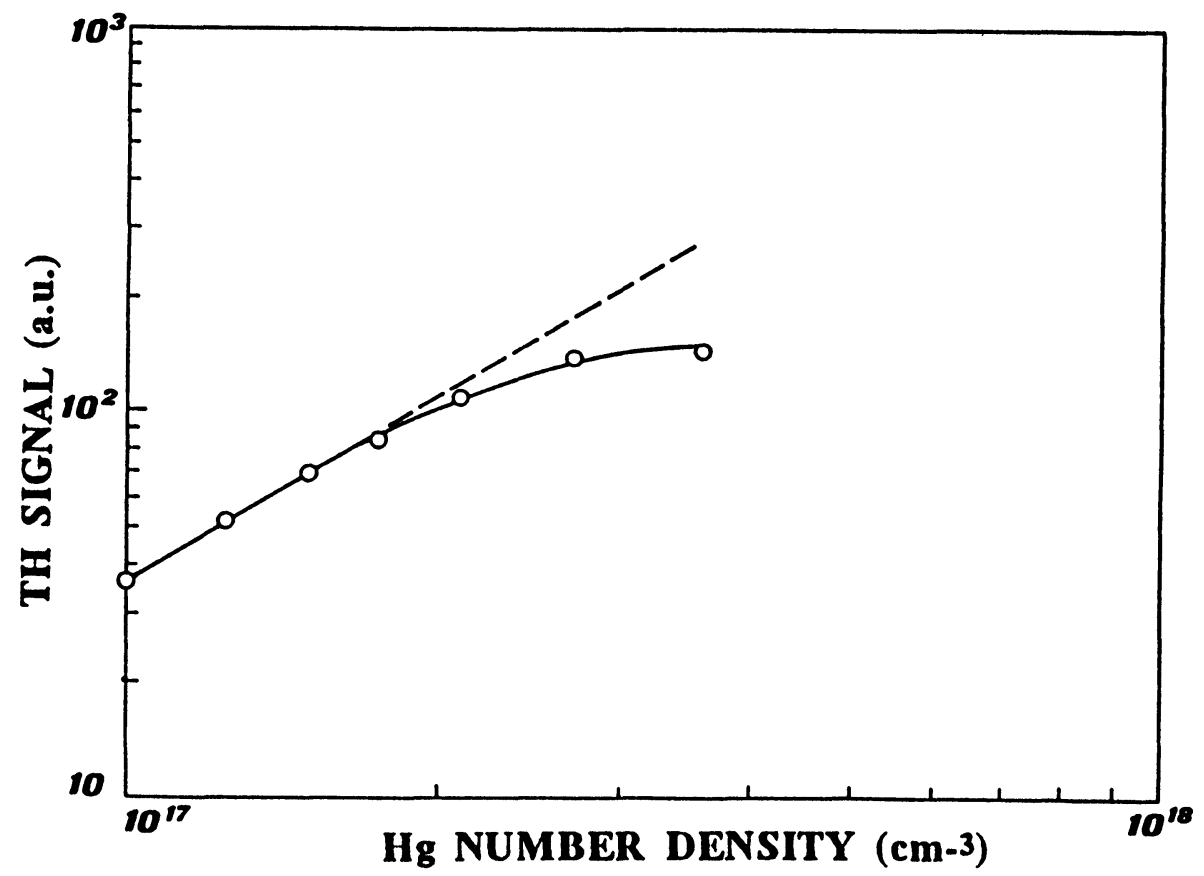

Figure $3 \mathrm{TH}$ intensity as a function of the $\mathrm{Hg}$ number density. The incoming intensity is $\mathrm{I}=5.8$ $10^{8} \mathrm{~W} / \mathrm{cm}^{2}$.

coefficient $(\alpha=\sigma \mathrm{N})$ at $268.8 \mathrm{~nm}$ is approximately $\mathrm{N}^{2}\left(710^{-40}\right) \mathrm{cm}^{-1}$. For the maximum $\mathrm{Hg}$ number density in use, $3.510^{17} \mathrm{~cm}^{-3}$, the absorption coefficient at the fundamental wavelength is $910^{-5} \mathrm{~cm}^{-1}$. The absorption coefficient at the harmonic wavelength for the lowest number density in use is approximately $0.04 \mathrm{~cm}^{-1}$, (estimated using photoionization data reported by $\mathrm{Brehm},{ }^{20}$ and the cross-section values reported by Berkowit $\mathrm{z}^{18}$ ). Consequently the absorption at the harmonic wavelength dominates, and the depletion of the fundamental beam due to one photon absorption can be neglected. In our case, where the active medium length $\mathrm{L}=10 \mathrm{~cm}$, the confocal parameter $b=2.7 \mathrm{~cm}$, and the focusing is at the middle of the medium, the absorption of the propagated $\mathrm{TH}$ reaches $50 \%$ at a number density of $\mathrm{N}=3.5$ $10^{17} \mathrm{~cm}^{-3}$. At these densities an additional increase of the absorption at the harmonic wavelength is expected from the $\mathrm{Hg}$ dimers ${ }^{29}$ which have a small peak near $89.6 \mathrm{~nm}$ (at $202^{\circ} \mathrm{C}$ the $\mathrm{Hg}_{2}$ number density is approximately ${ }^{30} 2.510^{12} \mathrm{~cm}^{-3}$ ). The conclusion is that the existence of saturation in the experimental results of Figure 3 could be justified by the absorption of the $\mathrm{TH}$, but the position where the saturation starts is far from the one indicating by the theory. ${ }^{16}$ As we will show below, the population transfer to the two photon resonant state can rectify the disagreement.

Figure 4 shows the intensity of the TH signal as a function of the He number density, for three $\mathrm{Hg}$ number densities. The He gas is affecting the phase-matching and the maximum TH signal appears at higher He number densities with increased $\mathrm{Hg}$ pressure. Furthermore as the $\mathrm{Hg}$ number density increases, the effect of $\mathrm{He}$ is 


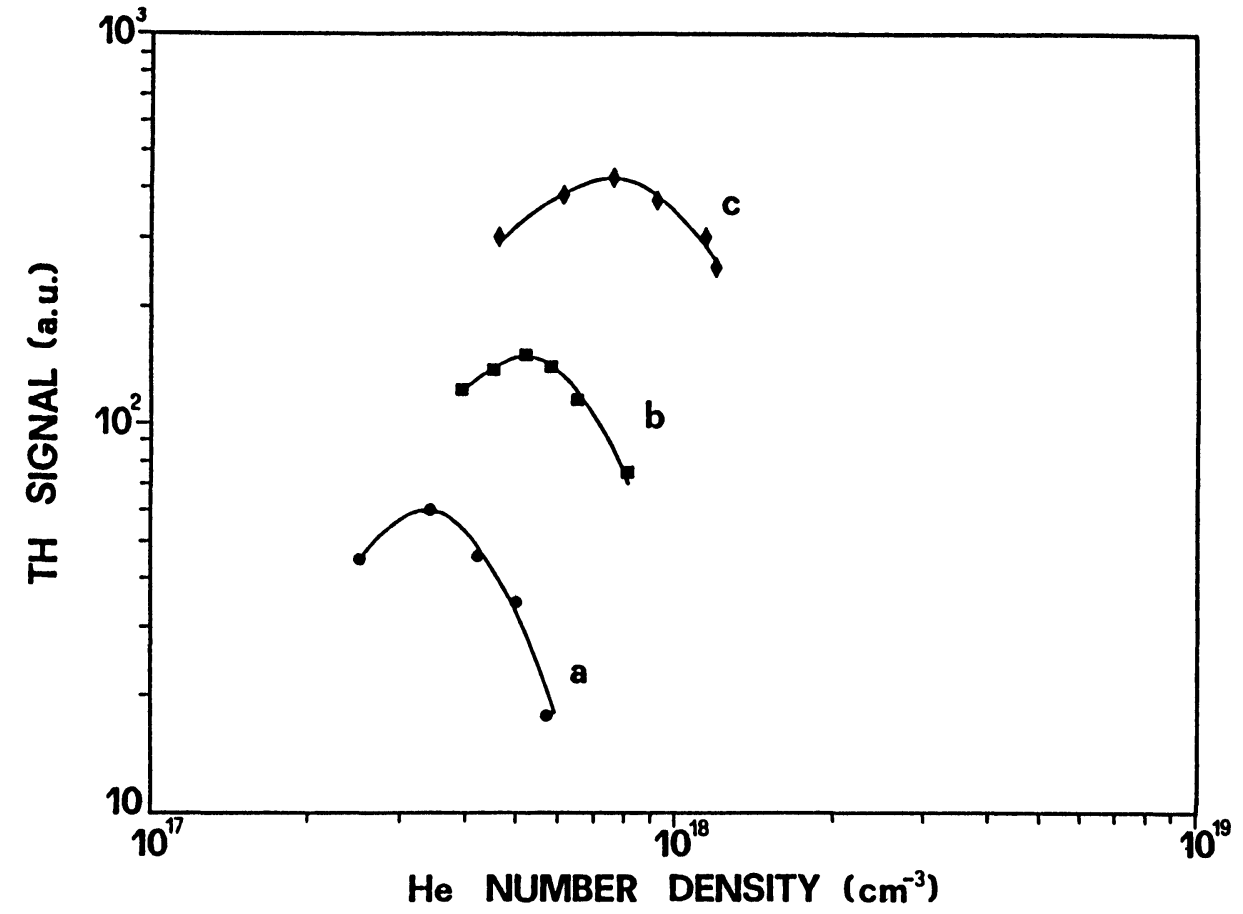

Figure 4 TH intensity as a function of the $\mathrm{He}$ number density for different $\mathrm{Hg}$ number densities: a) 8 $10^{16} \mathrm{~cm}^{-3}$, b) $1.410^{17} \mathrm{~cm}^{-3}$, c) $3.510^{17} \mathrm{~cm}^{-3}$. The incoming intensity is $\mathrm{I}=5.810^{8} \mathrm{~W} / \mathrm{cm}^{2}$.

weaker. It was mentioned above that $\mathrm{C}_{\mathrm{Hg}}=-3.6910^{-17} \mathrm{~cm}^{2}$, while $\mathrm{C}_{\mathrm{He}}=3.68$ $10^{-19} \mathrm{~cm}^{2}$, two orders of magnitude less. Consequently, for the case b of Figure 4 and according to the calculations presented by Bjorklund, ${ }^{16}$ maximization of the function $\mathrm{F}$ and the third harmonic signal should be expected at a He number density of $1.210^{19} \mathrm{~cm}^{-3}$. This is in disagreement with the experimental results of Figure 4.

The disagreements between the theoretical and the experimental results of Figure 3 and Figure 4, concerning the phase matching, require a modification of the $\Delta \mathrm{k}$ value. Some processes that can change the value of $\Delta \mathrm{k}$ are the excited state population (caused by two-photon absorption), the ionization and the AC-Stark shift, and their effects will be studied in subsequent sections.

Figure 5 shows the intensity of the TH signal as a function of the incident beam intensity for $\mathrm{N}=1.410^{17} \mathrm{~cm}^{-3}$, a value which is below saturation. For intensities below $410^{8} \mathrm{~W} / \mathrm{cm}^{2}$ the dependence is nearly cubic as expected. For higher intensities saturation appears, which indicates the existence of the processes mentioned above, which are limiting ones. The effect of such processes on the TH generation has been also considered by Georges et al, ${ }^{6,7}$ Ward et al, ${ }^{31}$ Wallace et al, ${ }^{32}$ and Junginger et $\mathrm{al},{ }^{33}$ and it will be discussed in the following sections.

Figure 6 shows the FWHM linewidth square of the two photon resonance as a function of the incident beam intensity square. The linewidth was measured by recording the $\mathrm{TH}$ signal as a function of the $2 \omega_{1}$ photons energy. The dependence indicates that power broadening exists. ${ }^{34}$ At intensities higher than $610^{8} \mathrm{~W} / \mathrm{cm}^{2}$ the 


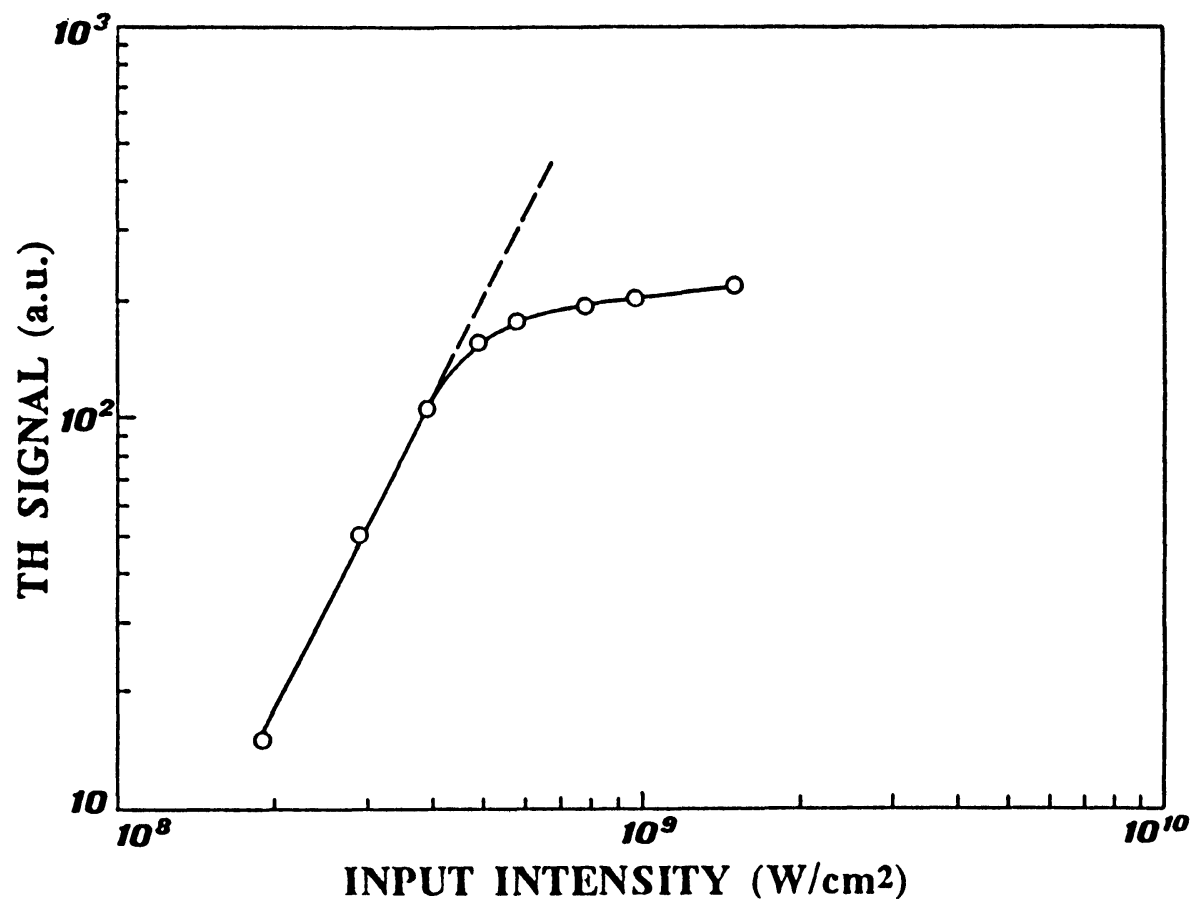

Figure $5 \mathrm{TH}$ intensity as a function of the input laser intensity. The number densities are $\mathrm{N}_{\mathrm{Hg}}=1.4$ $10^{17} \mathrm{~cm}^{-3}$ and $\mathrm{N}_{\mathrm{He}}=5.210^{17} \mathrm{~cm}^{-3}$.

slope increases. This can be attributed to additional broadening processes such as exponential growth of ASE from the $8^{1} \mathrm{~S}_{0}$ state, populated by two-photon absorption process, to lower $\mathrm{P}_{1}$ states, and AC-Stark broadening. The zero intensity linewidth is approximately $1.3 \mathrm{~cm}^{-1}$ (estimated using Figure 6) a value larger than the Doppler broadening and the laser linewidth, which are approximately $0.1 \mathrm{~cm}^{-1}$. Consequently the collision broadening dominates the process.

\subsection{Contribution of the Population Transfer to $\Delta k$.}

Calculation of the two-photon excitation rate was carried out using an equation reported by Stappaerts. ${ }^{8}$ Maximum contribution to the rate is expected to come from the $6^{3} \mathrm{P}_{1}$ state, because the $\omega_{1}$ photon is in near resonance, the $6^{1} \mathrm{P}_{1}$ state, because it has the maximum matrix element from all $6^{1} \mathrm{~S}_{0}-n \mathrm{P}_{1}$ transitions, and the $7^{1} \mathrm{P}_{1}, 8^{1} \mathrm{P}_{1}$ states, because they have the maximum matrix elements from all the $8^{1} \mathrm{~S}_{0}-\mathrm{nP}_{1}$ transitions. These matrix elements, were estimated using the corresponding oscillator strength values, which were calculated using emission transition probabilities reported by Hafner et al. ${ }^{35}$ The calculated oscillator strengths and the corresponding matrix elements are presented in Table 3 . The sign of the matrix elements was determined using the formalism reported by Bates et al, ${ }^{36}$ with a correction reported by Bebb. ${ }^{37}$ The calculated two-photon excitation rate $\mathrm{W}^{(2)}$ is approximately $1.310^{-10} \mathrm{I}^{2} \mathrm{sec}^{-1}$, where $I$ is in W/cm $\mathrm{cm}^{2}$, for on resonance transition and a linewidth of $1.3 \mathrm{~cm}^{-1}$ (Figure 6 ). The two-photon excitation $\mathrm{W}^{(2)} \tau$, where $\tau$ is the shortest time of the laser pulse 


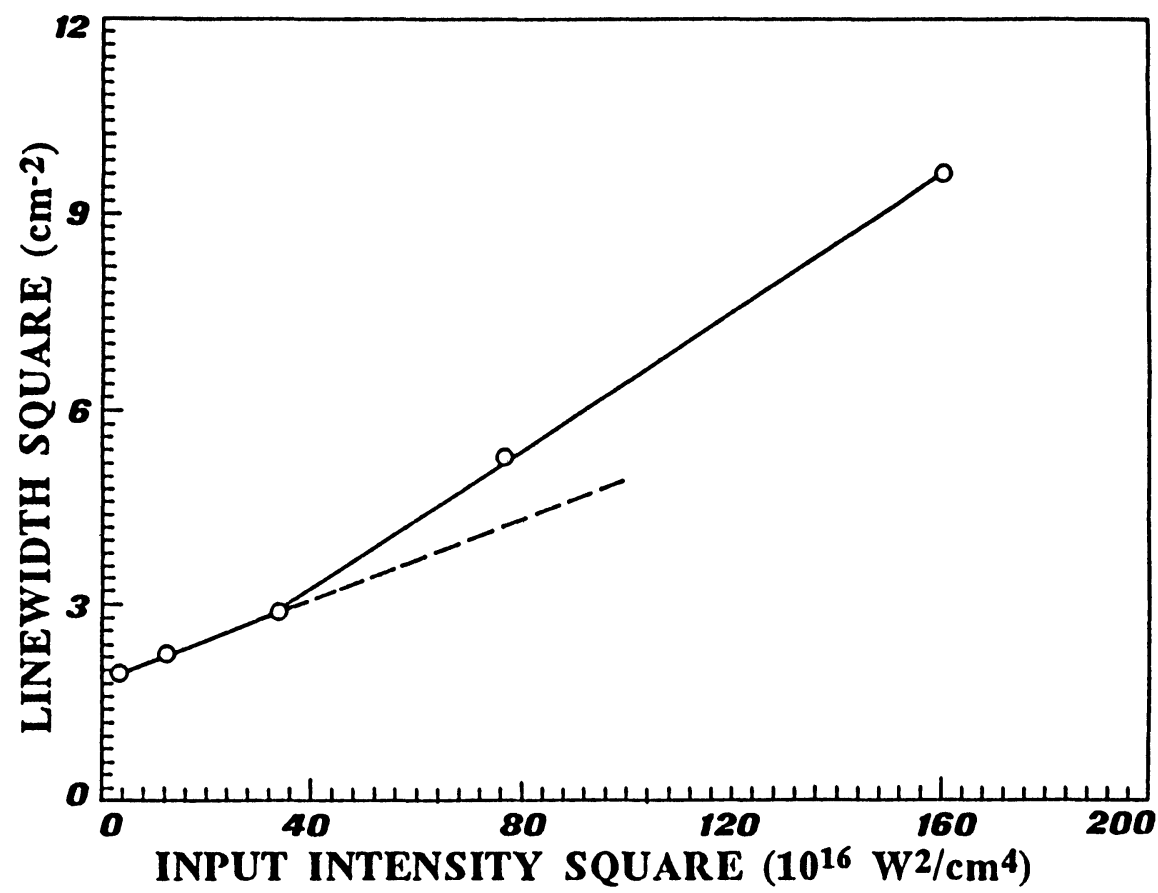

Figure 6 Two-photon resonance FWHM linewidth square as a function of the input laser intensity square. The number densities are $\mathrm{N}_{\mathrm{Hg}}=1.410^{17} \mathrm{~cm}^{-3}$ and $\mathrm{N}_{\mathrm{He}}=5.210^{17} \mathrm{~cm}-3$.

$(17 \mathrm{~ns})$ or the natural lifetime ${ }^{8}$ of the $8^{1} \mathrm{~S}_{0}$ state which is $84 \mathrm{nsec},{ }^{35}$ is being saturated (50\% population transfer) during the laser pulse for an intensity of $4.810^{8} \mathrm{~W} / \mathrm{cm}^{2}$ (at the focal plane). The net conclusion then is that in the case of Figure 4 (I = 5.8 $10^{8} \mathrm{~W} / \mathrm{cm}^{2}$ ) the population transfer to the $8^{1} \mathrm{~S}_{0}$ state is considerable and its effect on $\Delta \mathrm{k}$ should be included.

Table 3 Oscillator strengths for the transitions $8^{1} \mathrm{~S}_{0}-\mathrm{nP}_{1}, \mathrm{n}=6,7,8$, estimated using data given by Hafner and Schwarz, ${ }^{35}$ matrix elements for the same transitions and energy level spacings given by Moore. ${ }^{19}$

\begin{tabular}{llllr}
\hline Transition & $\begin{array}{l}\text { Energy } \\
\text { spacing } \text { cm }^{-1}\end{array}$ & $\begin{array}{l}\text { Emission } \\
\text { probability } 108 \\
\text { sec }^{-1}\end{array}$ & $f$ & $r 10^{-9} \mathrm{~cm}$ \\
\hline $8^{1} \mathrm{~S}_{0}-6^{3} \mathrm{P}_{1}$ & -35008 & 0.0045 & -0.0006 & 0.230 \\
$8^{1} \mathrm{~S}_{0}-6^{1} \mathrm{P}_{1}$ & -20336 & 0.0790 & -0.0286 & 2.078 \\
$8^{1} \mathrm{~S}_{0}-7^{3} \mathrm{P}_{1}$ & -4743 & 0.0051 & -0.0340 & 4.693 \\
$8^{1} \mathrm{~S}_{0}-7^{1} \mathrm{P}_{1}$ & -3109 & 0.0660 & -1.0232 & 31.808 \\
$8^{1} \mathrm{~S}_{0}-8^{3} \mathrm{P}_{1}$ & 2063 & 0.0010 & 0.1057 & -12.543 \\
$8^{1} \mathrm{~S}_{0}-8^{1} \mathrm{P}_{1}$ & 2459 & 0.0190 & 1.4136 & -42.024 \\
\hline
\end{tabular}

For the calculation of the $8^{1} \mathrm{~S}_{0}$ state contribution to $\Delta \mathrm{k}$ we need to know the matrix elements of the transitions $8^{1} \mathrm{~S}_{0}-\mathrm{nP}_{1}$. To our knowledge there are no data for these 
matrix elements except for the emission transition probabilities of the transitions ${ }^{1} S_{0}-n^{1,3} P_{1}$ where $n=6,7,8$, reported by Hafner et al, ${ }^{35}$ and presented in Table 3, but reasonable estimates can be made. In our case, the TH starting from the ${ }^{1} \mathrm{~S}_{0}$ reaches far in the continuum, and consequently its contribution to the excited state $\Delta \mathrm{k}$ is much lower than the contribution of the fundamental radiation, which is near resonant to some states both below and above the ionization limit. Consequently the TH contribution will be neglected.

For the ${ }^{1} \mathrm{~S}_{0}$ atoms, the largest contribution to the wavevector mismatch, at the fundamental wavelength, comes from the $6^{3} \mathrm{P}_{1}$ state because it corresponds to a near resonant transition, and the $6^{1} \mathrm{P}_{1}, 7^{1,3} \mathrm{P}_{1}, 8^{1,3} \mathrm{P}_{1}$ states because they have large matrix elements. Using data from Table 3 the contribution to the wavevector mismatch is calculated to be approximately $10^{-17} \mathrm{~N}_{2} \mathrm{~cm}^{-1}$, where $\mathrm{N}_{2}$ is the population of the excited state. This value is positive and consequently alters the ground state contribution to lower negative values. For example, for an input intensity of 5.8 $10^{8} \mathrm{~W} / \mathrm{cm}^{2}$ (at the focal plane) and a population transfer of 50\%, which is the case (b) curve in Figure 4, the excited state contribution to $\Delta \mathrm{k}$, reduces the wavevector mismatch to $\Delta \mathrm{k}_{\mathrm{Hg}}=-2.6 \mathrm{~cm}^{-1}$. The He contribution for the pressures used in this case is approximately $\Delta \mathrm{k}_{\mathrm{He}}=0.2 \mathrm{~cm}^{-1}$. Consequently the phase mismatch at the maximum of the case $\mathrm{b}$ in Figure 4, is $\Delta \mathrm{k}=-2.4 \mathrm{~cm}^{-1}$, a value which is still away from the optimum one $\Delta \mathrm{k}_{\mathrm{opt}}=-2 / \mathrm{b}=-0.75 \mathrm{~cm}^{-1}$.

So far, in the calculation of $8^{1} \mathrm{~S}_{0}$ atoms contribution to the wavevector mismatch, we have not included contributions coming from other bound or autoionizing states, because no information is available about the oscillator strengths of the necessary transitions. There is still a possibility to investigate their influence on the $\Delta \mathrm{k}$ value if we make some reasonable assumptions. Comparing the values of the oscillator strength for the transitions ${ }^{1} \mathrm{~S}_{0}-6 \mathrm{p}^{\prime}{ }^{3} \mathrm{P}_{1}(\mathrm{f}=0.53$, from Table 3$)$ and ${ }^{1} \mathrm{~S}_{0}-6 \mathrm{p}^{\prime}{ }^{3} \mathrm{P}_{1}$ ( $f=0.061$, reported by Alford and $\mathrm{Smith}^{38}$ ) we can see that the oscillator strength for transition starting from an excited state is lower by one order of magnitude than in the case starting from the ground state. Assuming that the same relation exists in the case for transitions $8^{1} \mathrm{~S}_{0}$-autoionizing state (the values of the oscillator strength for transitions $8^{1} \mathrm{~S}_{0}$-autoionizing state are expected to be even less), the contribution of the first four autoionizing states is estimated to be approximately $10^{-18} \mathrm{~N}_{2} \mathrm{~cm}^{-1}$ and it can be neglected. Furthermore, comparing the values of the oscillator strength for transitions $6^{1} \mathrm{~S}_{0}-\mathrm{nP}_{1}$ (Table 1) and ${ }^{1} \mathrm{~S}_{0}-\mathrm{nP}_{1}$ (Alford, Smith ${ }^{38}$ ), oscillator strengths for transitions starting from an excited $S_{0}$ state are less than the ones from the ground state. Assuming oscillator strengths for transitions $8^{1} \mathrm{~S}_{0}-n \mathrm{P}_{1}$ with $\mathrm{n}>9$ as high as the ones from the ground state their contribution is calculated to be $10^{-19} \mathrm{~N}_{2} \mathrm{~cm}^{-1}$ and it can be neglected also. However, the remaining transitions $8^{1} \mathrm{~S}_{0}-6 \mathrm{p}^{\prime}{ }^{1} \mathrm{P}_{1}, 8^{1} \mathrm{~S}_{0}-$ $9^{1} \mathrm{P}_{1}$ are expected to give considerable contribution to the excited state wavevector mismatch. As it can be seen, using the data from Table 1 and the values of the oscillator strength for the transitions $7^{1} \mathrm{~S}_{0}-6 \mathrm{p}^{\prime}{ }^{1} \mathrm{P}_{1}, 7^{1} \mathrm{~S}_{0}-9^{1} \mathrm{P}_{1}$ reported by Alford and Smith, ${ }^{38}$ the oscillator strengths for transitions to the $6 \mathrm{p}^{\prime}{ }^{1} \mathrm{P}_{1}, 9^{1} \mathrm{P}_{1}$ states are greater or equal to the ones for transitions to the $8^{1} \mathrm{P}_{1}$ state. This is probably due to the presence of the $6 \mathrm{p}^{\prime}{ }^{1} \mathrm{P}_{1}$ state, and is very important for our calculation because the oscillator strength for the transition $8^{1} \mathrm{~S}_{0}-8^{1} \mathrm{P}_{1}$ is the largest one of all $8^{1} \mathrm{~S}_{0}-\mathrm{nP}_{1}$ 
transitions. If we assume that the transitions $8^{1} S_{0}-6 p^{\prime}{ }^{1} P_{1}, 8^{1} S_{0}-9^{1} P_{1}$ have oscillator strengths one ordet of magnitude less than the one of the $8^{1} \mathrm{~S}_{0}-8^{1} \mathrm{P}_{1}$ transition, the $8^{1} \mathrm{~S}_{0}$ atoms contribution to the wavevector mismatch increases to $210^{-17} \mathrm{~N}_{2} \mathrm{~cm}^{-1}$. Then, in the case (b) of Figure $4, \Delta \mathrm{k}$ is approximately $\Delta \mathrm{k}_{\mathrm{Hg}}=-1.2 \mathrm{~cm}^{-1}$. Consequently, the $\mathrm{He}$ buffer gas can affect the phase-matching, which is clearly demonstrated in Figure 4, and the wavevector mismatch at the maximum is $\Delta \mathrm{k}=1$ $\mathrm{cm}^{-1}$, a value which is very near to the optimum one $\Delta \mathrm{k}_{\mathrm{opt}}=-2 / \mathrm{b}=-0.75 \mathrm{~cm}^{-1}$. This proves that our assumptions are correct.

\subsection{The effect of AC-Stark Shift, and the Ionization on the Phase-Mismatch}

So far we have not considered any AC-Stark shift. Only the fundamental radiation contributes to the shift as the harmonic intensity is orders of magnitude less and its wavelength is away from one of any transition. The relative shift of the ground and the two-photon resonance states due to the fundamental radiation can be calculated using a formula reported by Georges et $a^{16,7}$ and data from Tables 1 and 3. The calculated relative AC-Stark shift for the ${ }^{1} \mathrm{~S}_{0}, 8^{1} \mathrm{~S}_{0}$ states is given by $\delta V_{21}\left(\mathrm{~cm}^{-1}\right)=$ $10^{-10} \mathrm{I}\left(\mathrm{W} / \mathrm{cm}^{2}\right)$.

For the case of Figure $4\left(\mathrm{I}=5.810^{8} \mathrm{~W} / \mathrm{cm}^{2}\right)$ the calculated shift is approximately $0.06 \mathrm{~cm}^{-1}$ (at the focal plane) which is lower than the laser and the state linewidths. Consequently it has no effect on the processes. However at higher intensities, the shift can cause enough detuning of the $8^{1} \mathrm{~S}_{0}$ state and introduce non-linearities to the refractive index, which will affect the phase matching.

As it was mentioned before, the generated TH lies in the continuum and consequently $\mathrm{Hg}$ atoms can be ionized, a process which causes a decrease in the efficiency, and contributes to $\Delta \mathrm{k}$. Electrons are created by photoionization through absorption of the TH by the ground state atoms, and absorption of the fundamental radiation by the $8^{1} \mathrm{~S}_{0}$ state atoms. Furthermore, ionization of the excited state population can occur by other emitted radiation lines such as parametric and ASE. However the ionization of the $8^{1} \mathrm{~S}_{0}$ state atoms by the fundamental radiation is expected to be dominant because any generated radiation is orders of magnitude weaker than the fundamental one. The refractive index at wavelength $\lambda$, due to a number of free electrons $\mathrm{N}_{\mathrm{e}}$ is given by the equation $\mathrm{n}_{e}-1=-\left(\mathrm{N}_{\mathrm{e}} \mathrm{e}^{2} \lambda^{2}\right) /\left(2 \pi \mathrm{m}_{\mathrm{e}} \mathrm{c}^{2}\right)$, reported by Reintjes. ${ }^{17}$ The electron contribution to $\Delta \mathrm{k}$, for $\mathrm{TH}$ generation at $89.6 \mathrm{~nm}$, is approximately $210^{-17} \mathrm{~N}_{\mathrm{e}} \mathrm{cm}^{-1}$, where $\mathrm{N}_{\mathrm{e}}$ in $\mathrm{cm}^{-3}$. Consequently, the wavevector mismatch can be affected only by a large enough value of $\mathrm{N}_{\mathrm{e}}$.

Experiments were carried out concerning the ionization of $\mathrm{Hg}$ during the $\mathrm{TH}$ generation. The maximum ionization coincides with the maximum TH signal, verifying the resonance condition. At intensities below $410^{8} \mathrm{~W} / \mathrm{cm}^{2}$ the dependence of ionization as a function of the input laser intensity is nearly quadratic, while at higher intensities it is linear, indicating that the two-photon excitation is saturated. At input intensity $5.810^{8} \mathrm{~W} / \mathrm{cm}^{2}$, and $\mathrm{Hg}$ number density $1.410^{17} \mathrm{~cm}^{-3}$, which is the case (b) of Figure 4, the measured number of electrons was approximately $10^{13} \mathrm{~cm}^{-3}$. Their contribution to the wavevector mismatch is $210^{-4} \mathrm{~cm}^{-1}$, which is too small with respect to the $\mathrm{Hg}$ contribution and can be neglected. 
In conclusion both the AC-Stark shift and the ionization have negligible contribution to the wavevector mismatch in this particular case, but the contribution to $\Delta \mathrm{k}$ of the population transfer is very important. Our calculation, under reasonable assumptions concerning transitions from the $8^{1} S_{0}$ state, has shown that the population transfer reduces the wavevector mismatch $\Delta \mathrm{k}_{\mathrm{Hg}}$, so that the He contribution $\Delta \mathrm{k}_{\mathrm{He}}$ can be effective.

\section{CONCLUSIONS}

We have shown that, intense THG is possible using moderate input intensity $\left(10^{8}-10^{9}\right.$ $\mathrm{W} / \mathrm{cm}^{2}$ ) with a conversion efficiency of $10^{-7}$. Tunability of the TH can be achieved over a short region of $5 \mathrm{~cm}^{-1}$ at high input intensity. Phase-matching by the use of a buffer gas can improve the efficiency of the produced radiation. Moderate increase of the $\mathrm{Hg}$ vapor can also increase the efficiency. However, there are several problems associated with the attempt to further improve the efficiency by increasing the $\mathrm{Hg}$ number density and the input intensity. In particular, increased number density causes saturation (due to absorption of TH and the phase matching) and the input beam causes population transfer to the two-photon resonant state, which at lower intensities contributes in a positive way to the wavevector mismatch resulting in improved efficiency. But at the same time the two-photon excitation causes ionization and other parametric processes which limit the efficiency by providing other channels competing with the desired THG. Also the AC-Stark shift can play a significant role at very high input intensities as it can introduce non-linearities in the index of refraction which affect the phase matching. The ionization of the medium is another loss mechanism which can affect the wavevector mismatch at much higher intensities.

Finally, it was shown for the first time under quite reasonable assumptions that the contribution to the wavevector mismatch of the population of the $8^{1} S_{0}$ excited state is very important and reduces the $\mathrm{Hg}$ ground state contribution to lower values, which can be influenced by the He gas. These results agree reasonably well with our experimental observations.

\section{Acknowledgements}

We thank Professor A.T. Georges for many helpful discussions. One of us (E.K.) would like to thank the FORTH/IESL for a graduate student scholarship.

\section{References}

1. K. M. Leung, J. F. Ward and B. J. Orr. Phys. Rev. A, 9, 2440 (1974).

2. H. Puell, K. Spanner, W. Falkenstein, W. Kaiser, and C. R. Vidal. Phys. Rev. A, 14, 2240 (1976).

3. R. Hilbig and R. Wallenstein. IEEE J. Quant. Elect., QE-17, 1566 (1981).

4. P. R. Herman and B. P. Stoichef. Optics Lett., 10, 502 (1985).

5. R. B. Miles, and S. E. Harris. IEEE J. Quant. Elect., QE-9, 470 (1973).

6. A. T. Georges, P. Lambropoulos, and J. H. Marburger. Phys. Rev. A, 15, 300 (1977).

7. J. C. Diels and A. T. Georges. Phys. Rev. A, 19, 1589 (1979). 
8. E. A. Stappaerts. IEEE J. Quant. Elect., QE-15, 110 (1979).

9. H. Puell, H. Scheingraber and C. R. Vidal. Phys. Rev. A, 22, 1165 (1980).

10. H. Scheingraber and C. R. Vidal. Optics Com., 38, 75 (1981).

11. H. Scheingraber and C. R. Vidal. IEEE J. Quant. Elect., QE-19, 1747 (1983).

12. A. V. Smith, W. J. Alford and G. R. Hadley. J. Opt. Soc. Am. B, 5, 1503 (1988).

13. J. Bokor, R. R. Freeman, R. L. Panock and J. C. White. Optics Lett., 6, 182 (1981).

14. R. Mahon, and F. S. Tomkins. IEEE J. Quant. Electr., QE-18, 913 (1982).

15. P. P. Sorokin, J. J. Wynne, J. A. Armstrong and R. T. Hodgson. Annals N.Y Acad Sci., 267, 30 (1976).

16. G. C. Bjorklund. IEEE J. Quant. Elect., QE-11, 287 (1975).

17. J. F. Reintjes. Nonlinear optical parametric processes in liquids and gases (Academic Press, 1984), p. 50 and p. 227.

18. J. Berkowitz. Photoabsorption, Photoionization and Photoelectron Spectroscopy (Acad. Press, 1979), p. 90.

19. C. E. Moore. Atomic energy levels, (Ed. USA Dep. of Com., Nat. Bur. Stan., 1971), Vol. 3, p. 191.

20. B. Brehm. Z. Naturforsch, 21a, 196 (1966).

21. T. B. Lucatorto, T. J. Mcllrath and J. R. Roberts. Applied Optics, 18, 2505 (1979).

22. N. J. Dovichi, D. S. Moore and R. A. Keller. Applied Optics, 21, 1468 (1982).

23. A. V. Smith and W. J. Alford. Phys. Rev. A, 23, 3172 (1985).

24. G. Alber and P. Zoller. Phys. Rev. A, 27, 1373 (1982).

25. R. Lincke and B. Stredele. Z. Physik, 238, 164 (1970).

26. F. Schäfers, G. Schönhense and U. Heinzmann. Z. Phys. A; Atoms and Nuclei, 304, 41 (1982).

27. J. D. Hey, H. S. T. Driver and D. B. Fish. Amer. J. Phys., 56, 646 (1988).

28. R. E. Drullinger, M. M. Hessel and E. W. Smith. J. Chem. Phys., 66, 5656 (1977).

29. S. H. Linn, C. L. Liao, C. X. Liao, J. M. Brom Jr and C. Y. Ng. Chem. Phys. Lett., 105, 645, (1984).

30. K. Hilpert. J. Chem. Phys., 77, 1425 (1982).

31. J. F. Ward and A. V. Smith. Phys. Rev. Lett., 35, 653 (1975).

32. S. C. Wallace and G. Zdasiuk. Appl. Phys. Lett., 28, 449 (1975).

33. H. Junginger, H. B. Puell, H. Scheingraber and C. R. Vidal. IEEE J. Quant. Elect., QE-16, 1132 (1980).

34. C. C. Wang and L. I. Davis Jr. Phys. Rev. Lett., 35, 650 (1975).

35. P. Hafner and W. H. E. Schwarz. J. Phys. B: Atom. Mol. Phys., 11, 2975 (1978).

36. D. R. Bates, and A. Damgaard. Phil. Trans. Roy. Soc. London, A242, 101 (1949).

37. H. B. Bebb. Phys. Rev., 149, 25 (1966).

38. W. J. Alford and A. V. Smith. Phys. Rev. A, 36, 641 (1987). 